\title{
Article
}

\section{A Characterization of GRW Spacetimes}

\author{
Ibrahim Al-Dayel ${ }^{1}\left(0\right.$, Sharief Deshmukh ${ }^{2, *}$ and Mohd. Danish Siddiqi ${ }^{3}(1)$ \\ 1 Department of Mathematics and Statistics, College of Science, Imam Mohammad Ibn Saud Islamic University, \\ P.O. Box 65892, Riyadh 11566, Saudi Arabia; iaaldayel@imamu.edu.sa \\ 2 Department of Mathematics, College of Science, King Saud University, P.O. Box 2455, \\ Riyadh 11451, Saudi Arabia \\ 3 Department of Mathematics, College of Science, Jazan University, Jazan 45142, Saudi Arabia; \\ msiddiqi@jazanu.edu.sa \\ * Correspondence: shariefd@ksu.edu.sa
}

check for

updates

Citation: Al-Dayel, I.; Deshmukh, S.; Siddiqi, M.D. A Characterization of GRW Spacetimes. Mathematics 2021, 9, 2209. https://doi.org/10.3390/ math9182209

Academic Editor: Juan De Dios Pérez

Received: 16 August 2021

Accepted: 24 August 2021

Published: 9 September 2021

Publisher's Note: MDPI stays neutral with regard to jurisdictional claims in published maps and institutional affiliations.

Copyright: (c) 2021 by the authors. Licensee MDPI, Basel, Switzerland. This article is an open access article distributed under the terms and conditions of the Creative Commons Attribution (CC BY) license (https:/ / creativecommons.org/licenses/by/ $4.0 /)$.

\begin{abstract}
We show presence a special torse-forming vector field (a particular form of torse-forming of a vector field) on generalized Robertson-Walker (GRW) spacetime, which is an eigenvector of the de Rham-Laplace operator. This paves the way to showing that the presence of a time-like special torseforming vector field $\xi$ with potential function $\rho$ on a Lorentzian manifold $(M, g), \operatorname{dim} M>5$, which is an eigenvector of the de Rham Laplace operator, gives a characterization of a GRW-spacetime. We show that if, in addition, the function $\xi(\rho)$ is nowhere zero, then the fibers of the GRW-spacetime are compact. Finally, we show that on a simply connected Lorentzian manifold $(M, g)$ that admits a time-like special torse-forming vector field $\xi$, there is a function $f$ called the associated function of $\xi$. It is shown that if a connected Lorentzian manifold $(M, g), \operatorname{dim} M>4$, admits a time-like special torseforming vector field $\xi$ with associated function $f$ nowhere zero and satisfies the Fischer-Marsden equation, then $(M, g)$ is a quasi-Einstein manifold.
\end{abstract}

Keywords: generalized Robertson-Walker spacetime; special torse-forming vector fields; de RhamLaplace operator; quasi-Einstein manifold

MSC: $83 \mathrm{~F} 05 ; 53 \mathrm{C} 25$

\section{Introduction}

It is well known that through cosmological considerations the space being homogeneous and isotropic in the large scale, picks the Robertson-Walker metrics. It amounts to the fact that an $n$-dimensional spacetime, $n>3$, acquires the form $I \times{ }_{\varphi} N$, with metric $g=-d t^{2}+\varphi^{2} \bar{g}$, where $I$ is an open interval, $\varphi$ is a smooth positive function defined on $I$, and $(N, \bar{g})$ is an $(n-1)$-dimensional Riemannian manifold of constant curvature. An $n$-dimensional generalized Robertson-Walker spacetime (GRW-spacetime) is $I \times{ }_{\varphi} N$, with metric $g=-d t^{2}+\varphi^{2} \bar{g}$, where $(N, \bar{g})$ is an $(n-1)$-dimensional Riemannian manifold (cf. [1,2]). An interesting characterization of GRW-spacetime was obtained by Chen (cf. [3]), by proving that a Lorentzian manifold $(M, g)$ admits a non-trivial time-like concircular vector field, if, and only if, it is a GRW-spacetime. Additionally, for interesting characterizations of GRW-spacetimes using torse-forming vector fields and Weyl tensors, we refer to (cf. $[4,5])$.

A concircular vector field $\xi$ on a semi-Riemannian manifold $(M, g)$ satisfies:

$$
\nabla_{U} \xi=\rho U, \quad U \in \mathfrak{X}(M),
$$

where $\rho$ is a scalar, $\nabla$ is a Levi-Civita connection, and $\mathfrak{X}(M)$ is the Lie algebra of smooth vector fields on $M$ (cf. [5-7]). For other characterizations of GRW-spacetimes, we refer to (cf. $[2,3,8,9])$. 
Yano generalized concircular vector fields by introducing a torse-forming vector field on semi-Riemannian manifold $(M, g)$ (cf. [10]), defined by:

$$
\nabla_{U} \xi=\rho U+\alpha(U) \xi, \quad U \in \mathfrak{X}(M),
$$

where $\alpha$ is a 1 -form called the torsed 1-form. Naturally, if $\alpha=0$, then a torse-forming vector field is a concircular vector field. These vector fields are also used in characterizing a GRW-spacetime (cf. [2,4]). In [11], Chen considered an interesting special class of torseforming vector field, requiring $\xi$ to be nowhere zero and satisfying $\alpha(\xi)=0$, that is the torse-forming vector field is perpendicular to the dual-vector field to torsed form $\alpha$, called torqued vector fields.

In the present paper, we introduce on a Lorentzian manifold a special type of torseforming vector field. A unit time-like torse-forming vector field $\xi$ on a Lorentzian manifold $(M, g)$ is said to be a special torse-forming vector field if it satisfies:

$$
\nabla_{U} \xi=\rho(U+\eta(U) \xi), \quad U \in \mathfrak{X}(M),
$$

where $\rho$ is a non-zero function and $\eta$ is 1 -form dual to $\xi$. We call $\rho$ the potential function of the special torse-forming vector field $\xi$. Note that for a special torse-forming vector field, using Equation (1), we have $\alpha(U)=-\rho \eta(U)$, that is $\xi$ is a torse-forming vector field, which is parallel to the vector field dual $\alpha$ as opposed to the torqued vector field where $\xi$ is orthogonal to the vector field dual $\alpha$. Moreover, from the definition of special torse-forming vector field $\xi$ on a Lorentzian manifold, it follows that under no situation, it reduces to a concircular vector field.

We study the role of a time-like special torse-forming vector field $\xi$ on a Lorentzian manifold $(M, g)$ in characterizing GRW-spacetimes. It is achieved by using the de RhamLaplace operator $\square$ (cf. [12]) and a time-like special torse-forming vector field $\xi$ with potential function $\rho$ on a connected Lorentzian manifold $(M, g), \operatorname{dim} M>5$, through showing that $\square \xi=\sigma \xi$ holds for a smooth function $\sigma$, if, and only if, $(M, g)$ is a GRWspacetime (see Theorem 1). We also show that if the function $\xi(\rho)$ is nowhere zero on $M$, then the fibers of GRW-spacetime $I \times{ }_{\varphi} N$ are compact (see Theorem 2).

If $\xi$ is a special torse-forming vector field on a simply connected Lorentzian manifold $(M, g)$, then the dual-1-form $\eta$ is closed (see Equation (15)), and, therefore, there is a function $f$ such that $\eta=d f$. Thus, the special torse-forming vector field $\xi$ on a simply connected Lorentzian manifold $(M, g)$ satisfies $\xi=\nabla f$, call this function $f$ the associated function of $\xi$. Recall that a Lorentzian manifold $(M, g)$ is said to be a quasi-Einstein manifold (cf. [13]) if its Ricci tensor has the following expression:

$$
\text { Ric }=f_{1} g+f_{2} \beta \otimes \beta,
$$

where $f_{1}, f_{2}$ are scalars and $\beta$ is a 1 -form on $M$. Exact solutions of the Einstein field equations can provide very important information about quasi-Einstein manifolds. For example, the Robertson-Walker spacetimes are quasi-Einstein manifolds. For this reason, the study of quasi-Einstein manifolds is important. It is shown that if the associated function $f$ of the special torse-forming vector field $\xi$ on a simply connected Lorentzian manifold $(M, g), \operatorname{dim} M>4$, satisfies (i) $f$ is nowhere zero and (ii) $f$ is a solution of the Fischer-Marsden equation, then $(M, g)$ is a quasi-Einstein manifold (see Theorem 3 ). Additionally, it is shown that if the scalar curvature $\tau$ of a simply connected Lorentzian manifold $(M, g), \operatorname{dim} M \geq 4$, is a constant and possesses a special torse-forming vector field $\xi$ with potential function $\rho$ and associated function $f$ satisfying the above two conditions, then the potential function $\rho$ is an eigenfunction of the Laplace operator $\Delta$ (see Corollary 1). 


\section{Preliminaries}

Let $\varphi$ be a smooth function on an $n$-dimensional connected Lorentzian $(M, g)$. The Hessian operator $H_{\varphi}$ is defined by:

$$
H_{\varphi}(V)=\nabla_{V} \nabla \varphi, \quad V \in \mathfrak{X}(M),
$$

where $\nabla \varphi$ is the gradient of $\varphi$ and $\operatorname{Hessian} \operatorname{Hess}(\varphi)$ is defined by (cf. [14]):

$$
\operatorname{Hess}(\varphi)\left(U_{1}, U_{2}\right)=g\left(H_{\varphi}\left(U_{1}\right), U_{2}\right), \quad U_{1}, U_{2} \in \mathfrak{X}(M) .
$$

The Laplacian $\Delta \varphi$ of the function $\varphi$ is given by $\Delta \varphi=\operatorname{div}(\nabla \varphi)$, and it satisfies:

$$
\Delta \varphi=\operatorname{tr} H_{\varphi} .
$$

Let $\xi$ be a time-like special torse-forming vector field on a Lorentzian $(M, g)$. Then, using the expression for the curvature tensor field

$$
R\left(F_{1}, F_{2}\right) F_{3}=\nabla_{F_{1}} \nabla_{F_{2}} F_{3}-\nabla_{F_{2}} \nabla_{F_{1}} F_{3}-\nabla_{\left[F_{1}, F_{2}\right]} F_{3}, \quad F_{1}, F_{2}, F_{3} \in \mathfrak{X}(M)
$$

and Equation (2), we compute:

$$
R\left(F_{1}, F_{2}\right) \xi=F_{1}(\rho) F_{2}-F_{2}(\rho) F_{1}+\left(F_{1}(\rho) \eta\left(F_{2}\right)-F_{2}(\rho) \eta\left(F_{1}\right)\right) \xi+\rho^{2}\left(\eta\left(F_{2}\right) F_{1}-\eta\left(F_{1}\right) F_{2}\right) .
$$
$(M, g)$ :

Above equation gives expression for the Ricci tensor Ric of the Lorentzian manifold

$$
\operatorname{Ric}(V, \xi)=-(n-4) V(\rho)+\left(\xi(\rho)+(n-3) \rho^{2}\right) \eta(V), \quad V \in \mathfrak{X}(M) .
$$

Note that the Ricci operator $Q$ of the Lorentzian manifold $(M, g)$ is given by $\operatorname{Ric}(U, V)=$ $g(Q U, V), U \in \mathfrak{X}(M)$, and, therefore, Equation (7) implies:

$$
Q \xi=-(n-4) \nabla \rho+\left(\xi(\rho)+(n-3) \rho^{2}\right) \xi
$$

and:

$$
\operatorname{Ric}(\xi, \xi)=-(n-3)\left(\xi(\rho)+\rho^{2}\right) .
$$

The Laplace operator $\Delta$ acting on vector fields on the Lorentzian manifold $(M, g)$ is defined by:

$$
\Delta U=\sum_{i=1}^{n}\left(\nabla_{v_{i}} \nabla_{v_{i}} U-\nabla_{\nabla_{v_{i}} v_{i}} U\right), \quad U \in \mathfrak{X}(M),
$$

where $\left\{v_{1}, \ldots, v_{n}\right\}$ is a local orthonormal frame on $M$. The de Rham-Laplace operator $\square$ on the Lorentzian manifold $(M, g)$ is $\square: \mathfrak{X}(M) \rightarrow \mathfrak{X}(M)$ given by (cf. [12]):

$$
\triangleright U=\Delta U+Q U, \quad U \in \mathfrak{X}(M) .
$$

Lemma 1. Let $\xi$ be a time-like special torse-forming vector on an n-dimensional Lorentzian manifold $(M, g)$ with potential function $\rho$. Then:

$$
\square \xi=-(n-5) \nabla \rho+2(n-2) \rho^{2} \xi .
$$

Proof. Using Equation (2), for $U \in \mathfrak{X}(M)$, we have:

$$
\nabla_{U} \nabla_{U} \xi-\nabla_{\nabla_{U} U} \xi=U(\rho) U+U(\rho) \eta(U) \xi+\rho^{2}\|U\|^{2} \xi+2 \rho^{2} \eta(U)^{2} \xi+\rho^{2} \eta(U) U .
$$


Since $\xi$ is a time-like unit vector field, choosing a local frame $\left\{v_{1}, \ldots, v_{n-1}, \xi\right\}$ on $M$, where $v_{i}, i=1, \ldots, n-1$ are spacelike unit vector fields in the above equation, to conclude:

$$
\Delta \xi=\nabla \rho-\left(\xi(\rho)-(n-1) \rho^{2}\right) \xi .
$$

Thus, using Equations (8) and (11) with the above equation, we conclude:

$$
\square \xi=-(n-5) \nabla \rho+2(n-2) \rho^{2} \xi .
$$

\section{Characterizing GRW Spacetimes}

Consider an n-dimensional GRW-spacetime $M=I \times{ }_{\varphi} N$ with metric $g=-d t^{2}+\varphi^{2} \bar{g}$. Then, $\xi=\frac{d}{d t}$ is a time-like unit vector field on $(M, g)$. Let $\nabla$ be the Levi-Civita connection on $(M, g)$. Then, for a $U \in \mathfrak{X}(M)$, we have $U=h \xi+E, E \in \mathfrak{X}(N)$. If we denote by $\eta=d t$, then $\eta(U)=g(U, \xi)=-h$, where $\eta(\xi)=g(\xi, \xi)=-1$. Using fundamental equations for the warped product (cf. [8]), we have:

$$
\nabla_{U} \xi=\nabla_{h \xi+E} \xi=\nabla_{E} \xi=\frac{\xi(\varphi)}{\varphi} E=\frac{\xi(\varphi)}{\varphi}(U-h \xi)=\frac{\xi(\varphi)}{\varphi}(U+\eta(U) \xi) .
$$

Thus,

$$
\nabla_{U} \xi=\rho(U+\eta(U) \xi), \quad U \in \mathfrak{X}(M), \quad \rho=\frac{\xi(\varphi)}{\varphi},
$$

this proves, $\xi$ is a special torse-forming vector field on the GRW-spacetime $(M, g)$. Now, using the expression for the Ricci tensor for the warped product $I \times_{\varphi} N$ (cf. [8]), we have:

$$
\operatorname{Ric}(\xi, E)=0, \quad E \in \mathfrak{X}(N),
$$

which implies $Q(\xi)=\lambda \xi$ for a smooth function $\lambda$ on $I$. Furthermore, choosing a local frame $\left\{v_{1}, \ldots, v_{n-1}\right\}$ on $N$, we have a local orthonormal frame $\left\{\xi_{1}, v_{1}, \ldots, v_{n-1}\right\}$ on $M$. Then, using Equation (12), we have $\nabla_{\xi} \xi=0, \nabla_{v_{i}} \xi=\rho v_{i}, v_{i}(\rho)=0$, and:

$$
\nabla_{v_{i}} \nabla_{v_{i}} \xi=\rho \nabla_{v_{i}} v_{i}, \nabla_{\xi} \nabla_{\xi} \xi=0 .
$$

Furthermore, using Equation (12), we have:

$$
\nabla_{\nabla_{v_{i}} v_{i}} \xi=\rho\left(\nabla_{v_{i}} v_{i}+g\left(\nabla_{v_{i}} v_{i}, \xi\right) \xi\right)=\rho \nabla_{v_{i}} v_{i}-\rho g\left(v_{i}, \nabla_{v_{i}} \xi\right) \xi=\rho \nabla_{v_{i}} v_{i}-\rho^{2} \xi .
$$

Thus, the rough Laplace operator $\Delta$ acting on $\xi$ is given by:

$$
\Delta \xi=\left(\nabla_{\xi} \nabla_{\xi} \xi-\nabla_{\nabla_{\xi} \xi} \xi\right)+\sum_{i=1}^{n-1}\left(\nabla_{v_{i}} \nabla_{v_{i}} \xi-\nabla_{\nabla_{v_{i}} u_{i}} \xi\right)=(n-1) \rho^{2} \xi .
$$

Now, we see that the de Rham-Laplace operator $\square$ acting on $\xi$ is given by:

$$
\nabla \xi=\left((n-1) \rho^{2}+\lambda\right) \xi .
$$

Hence, GRW-spacetime $(M, g)$ admits a special torse-forming vector field $\xi$, which is an eigenvector of the de Rham-Laplace operator $\square$.

Theorem 1. An n-dimensional connected Lorentzian manifold $(M, g), n>5$, is a GRW-spacetime $I \times{ }_{\varphi} N$, if, and only if, it admits a time-like special torse-forming vector field $\xi$, which is an eigenvector of the de Rham-Laplace operator on $(M, g)$. 
Proof. Let $(M, g)$ be a connected Lorentzian manifold, $n>5, \xi$ be a time-like special torse-forming vector field on $(M, g)$ with $\square \xi=\lambda \xi$, $\lambda$ being a scalar. We denote by $\nabla$ the Levi-Civita connection on $(M, g)$; using Equation (2), we have:

$$
\nabla_{\xi} \xi=0 .
$$

Define a smooth distribution $\mathcal{D}$ on $M$ by:

$$
\mathcal{D}=\{U \in \mathfrak{X}(M): \eta(U)=0\} .
$$

Note that Equation (2) gives:

$$
d \eta(U, V)=g\left(\nabla_{U} \xi, V\right)-g\left(\nabla_{V} \xi, U\right)=0, \quad U, V \in \mathfrak{X}(M),
$$

that is the dual-1-form $\eta$ to $\xi$ is closed. Thus, for $E, F \in \mathcal{D}$, we have $\eta([E, F])=-d \eta(E, F)=0$, that is $[E, F] \in \mathcal{D}$, proving that the distribution $\mathcal{D}$ is integrable. Let $N$ be a leaf of $\mathcal{D}$. Then, $N$ is a hypersurface of $M$ with unit normal $\xi$. Using Equation (2), we observe that for $E \in \mathfrak{X}(N)$,

$$
\nabla_{E} \xi=\rho E,
$$

that is the shape operator $S$ of $N$ is given by:

$$
S(E)=-\rho E, \quad E \in \mathfrak{X}(N) .
$$

Now, as $\square \xi=\lambda \xi$, where $\lambda$ is a scalar on $M$, using Lemma 1 , we get:

$$
-(n-5) \nabla \rho+2(n-2) \rho^{2} \xi=\lambda \xi .
$$

On taking the inner product in above equation with $\xi$ yields

$$
\lambda=(n-5) \xi(\rho)+2(n-2) \rho^{2}
$$

and substituting this value of $\lambda$ in Equation (18), we have:

$$
-(n-5) \nabla \rho=(n-5) \xi(\rho) \xi .
$$

Above equation on taking the inner product with $E \in \mathfrak{X}(N)$, gives $(n-5) E(\rho)=0$, and the assumption $n>5$ implies $E(\rho)=0$, that is $\rho$ is a constant on the hypersurface $N$. Therefore, Equation (17) implies that $N$ is a totally umbilical hypersurface of $M$. Moreover, the orthogonal complementary distribution $\mathcal{D}^{\perp}$ to $\mathcal{D}$ is one-dimensional spanned by $\xi$, and by Equation (13), the integral curves of the distribution $\mathcal{D}^{\perp}$ are geodesics on $M$. Thus, $(M, g)$ is the warped product $I \times_{\varphi} N$ (cf. [15]), that is $(M, g)$ is a GRW-spacetime.

Conversely, we have already seen that a GRW-spacetime $I \times_{\varphi} N$ admits a special torse-forming vector field $\xi$, which is an eigenvector of $\odot$.

In the above result we have seen that the presence of a time-like special torse-forming vector field $\xi$ on a Lorentzian manifold $(M, g)$ satisfying $\square \xi=\lambda \xi$ for scalar $\lambda$ is a GRWspacetime $I \times_{\varphi} N$. It is interesting to observe if in addition $\xi(\rho)$ is nowhere zero, then this condition has effect on the topology of $N$.

Theorem 2. Let $\xi$ be a time-like special torse-forming vector field with potential function $\rho$ on an $n$-dimensional complete and connected Lorentzian manifold $(M, g), n>5$. If $\xi$ is an eigenvector of the de Rham-Laplace operator on $(M, g)$ and the function $\xi(\rho)$ is nowhere zero, then $(M, g)$ is $G R W$-spacetime $I \times{ }_{\varphi} N$, with $N$ compact. 
Proof. Let $\xi$ be a time-like special torse-forming vector field on a Lorentzian manifold $(M, g), n>5$, with $\xi$ being an eigenvector of the de Rham Laplace operator on $(M, g)$ and the function $\xi(\rho) \neq 0$ everywhere on $M$. Since $n>5$, Equation (19) implies:

$$
\nabla \rho=-\xi(\rho) \xi
$$

As $\xi$ is a time-like unit vector field and $\xi(\rho)$ is nowhere zero, the above equation implies that $\nabla \rho$ is nowhere zero on $M$. Therefore, the potential function $\rho: M \rightarrow \mathbf{E}$ is a submersion, and each fiber $F_{x}=\rho^{-1}\{\rho(x)\}, x \in M$, is an $(n-1)$-dimensional smooth manifold; as $\{\rho(x)\}$ is compact in $\mathbf{E}$, we obtain that $F_{x}$ is compact. Consider a smooth vector field:

$$
\mathbf{u}=-\frac{\xi}{\xi(\rho)}
$$

that has no zeros on $M$. Then, it follows that $\mathbf{u}(\rho)=-1$ and $\mathbf{u}$ has a local flow $\left\{\phi_{s}\right\}$ that satisfies:

$$
\rho\left(\phi_{s}(x)\right)=\sigma(x)-s .
$$

Recall the escape Lemma (cf. [16]), which states that if $\gamma$ is a integral curve of $\mathbf{u}$ whose maximal domain is not all of $\mathbf{E}$, then the image of $\gamma$ cannot lie in any compact subset of $M$. Using the escape lemma and Equation (21) on a complete and connected $M$, we obtain that $\mathbf{u}$ is complete and has global flow $\left\{\phi_{s}\right\}$. Now, define $f: \mathbf{E} \times F_{x} \rightarrow M$ by:

$$
f(s, u)=\phi_{s}(u), \quad u \in F_{x} .
$$

Then, $f$ is smooth, and for each $u \in M$, we find $s \in \mathbf{E}$ such that $\phi_{s}(u)=y \in F_{x}$, satisfying $u=\phi_{-s}(y)$. Thus, $f(-s, y)=u$, that is $f$ is an on-to map. We observe that, on taking $\left(s_{1}, u_{1}\right),\left(s_{2}, v_{2}\right)$ in $\mathbf{E} \times F_{x}$ satisfying $f\left(s_{1}, u_{1}\right)=f\left(s_{2}, u_{2}\right)$, we have $\phi_{s_{1}}\left(u_{1}\right)=\phi_{s_{2}}\left(u_{2}\right)$, and using Equation (21), we obtain $\rho\left(u_{1}\right)-s_{1}=\rho\left(u_{2}\right)-s_{2}$. As $u_{1}, u_{2} \in F_{x}, \rho\left(u_{1}\right)=\rho\left(u_{2}\right)$, and we obtain $s_{1}=s_{2}$. Thus, using $\phi_{s_{1}}\left(u_{1}\right)=\phi_{s_{2}}\left(u_{2}\right)$, we arrive at $u_{1}=u_{2}$, that is $f$ is one-to-one. Furthermore, we have:

$$
f^{-1}(u)=(-s, y)=\left(-s, \phi_{s}(u)\right),
$$

which is smooth. Hence, $f: \mathbf{E} \times F_{x} \rightarrow M$ is a diffeomorphism, where $F_{x}$ is a compact subset of $M$. Using Theorem 3.1, we see that $I \times N$ is diffeomorphic to $\mathbf{E} \times F_{x}$, and as the open interval $I$ is diffeomorphic to $\mathbf{E}$, the fiber $N$ must be diffeomorphic to $F_{x}$. As $F_{x}$ is compact, we obtain that $N$ is compact.

\section{Lorentzian Manifolds as Quasi-Einstein Manifolds}

Fischer-Marsden considered the following differential equation on a semi-Riemannian manifold $(M, g)$ (cf. [17]):

$$
(\Delta f) g+f \operatorname{Ric}=\operatorname{Hess}(f),
$$

where $f$ is a smooth function on $M$. We call the above differential equation the FischerMarsden equation. This differential equation is closely associated with Einstein spaces. A generalization of Einstein manifolds was considered in [13], where the authors defined quasi-Einstein manifolds. A semi-Riemannian manifold $(M, g)$ is said to be a quasi-Einstein manifold if its Ricci tensor satisfies Equation (3). In this section, we use a unit time-like special torse-forming vector field $\xi$ on a Lorentzian manifold $(M, g)$ to find conditions under which $(M, g)$ is a quasi-Einstein manifold.

Let $\xi$ be a time-like special torse-forming vector field on a simply connected Lorentzian manifold $(M, g)$. On using Equations (2) and (15), we have $d \eta=0$, that is $\eta$ is a closed 1 -form and $M$ is simply connected $\eta=d f$ (exact) for a smooth function $f$ on $M$. Thus, for a time-like special torse-forming $\xi$ on a simply connected Lorentzian manifold $(M, g)$, we have:

$$
\xi=\nabla f
$$


and we call the smooth function $f$ in Equation (23) the associated function of $\xi$.

Theorem 3. Let $\xi$ be a time-like special torse-forming vector field on an n-dimensional simply connected Lorentzian manifold $(M, g), n>4$, with potential function $\rho$ and associated function $f$. If $f$ is a nowhere zero solution of the Fischer-Marsden equation, then $(M, g)$ is a quasi-Einstein manifold.

Proof. Using Equations (2) and (23), we have:

$$
H_{f}(U)=\rho(U+\eta(U) \xi)
$$

which implies:

$$
\operatorname{Hess}(f)=\rho g+\rho \eta \otimes \eta, \quad \Delta f=(n-3) \rho .
$$

Since $f$ satisfies Fischer-Marsden equation, using Equations (22) and (24), we have:

$$
f R i c=-(n-4) \rho g+\rho \eta \otimes \eta .
$$

As $f$ is nowhere zero, we have:

$$
\text { Ric }=-(n-4)\left(\rho f^{-1}\right) g+\left(\rho f^{-1}\right) \eta \otimes \eta .
$$

Hence, $(M, g)$ is a quasi-Einstein manifold.

If simply connected Lorentzian manifold $(M, g)$ has scalar curvature $\tau=\operatorname{tr} Q$, using above result we have the following result that gives a relation between $\rho$ and $f$ of the time-like special torse-forming vector field $\xi$ on $(M, g)$.

Corollary 1. Let $\xi$ be a time-like special torse-forming vector field on an n-dimensional simply connected Lorentzian manifold $(M, g), n \geq 4$, with potential function $\rho$ and associated function $f$. If $f$ is a solution of the Fischer-Marsden equation, then:

$$
\rho=-\frac{\tau}{(n-3)^{2}} f
$$

In particular, if the scalar curvature $\tau$ of $(M, g)$ is a constant, then the potential function $\rho$ is an eigenfunction of the Laplace operator $\Delta$.

Proof. Let $\xi$ be a time-like special torse-forming vector field on a simply connected Lorentzian manifold $(M, g), n \geq 4$, with potential function $\rho$ and associated function $f$. Suppose $f$ satisfies Equation (22). Then, Equation (25), gives

$$
f \tau=-(n-4)(n-2) \rho-\rho=-(n-3)^{2} \rho .
$$

Hence,

$$
\rho=-\frac{\tau}{(n-3)^{2}} f
$$

Now, if $\tau$ is a constant, then the above equation in view of Equation (24) implies:

$$
\Delta \rho=-\frac{\tau}{(n-3)} \rho,
$$

that is the potential function $\rho$ is an eigenfunction of $\Delta$.

Author Contributions: Conceptualization and methodology, I.A.-D., S.D. and M.D.S.; formal analysis, I.A.-D.; writing-original draft preparation, I.A.-D., S.D. and M.D.S.; writing-review and editing, S.D. and M.D.S.; supervision, S.D.; project administration I.A.-D. All authors read and agreed to the published version of the manuscript. 
Funding: The authors extend their appreciation to the Deanship of Scientific Research at Imam Mohammad Ibn Saud Islamic University for funding this through Research Group No. RG-21-09-09.

Institutional Review Board Statement: Not applicable.

Informed Consent Statement: Not applicable.

Data Availability Statement: Not applicable.

Conflicts of Interest: The authors declare no conflict of interest.

\section{References}

1. Mantica, C.A.; Molinari, L.G.; De, U.C. A note on generalized Robertson-Walker spacetimes. Int. J. Geom. Meth. Mod. Phys. 2016, 13, 1650079. [CrossRef]

2. Mantica, C.A.; Molinari, L.G. Generalized Robertson-Walker spacetimes-A survey. Int. J. Geom. Meth. Mod. Phys. 2017, 14, 1730001. [CrossRef]

3. Chen, B.-Y. A simple characterization of generalized Robertson-Walker spacetimes. Gen. Relativ. Gravit. 2014, 46, 1833-1835. [CrossRef]

4. Capozziello, S.; Mantica, C.A.; Molinari, L.G. Cosmological perfect fluids in higher-order gravity. Gen. Relativ. Gravit. 2020, 52, 36. [CrossRef]

5. Ishihara, S. On infinitesimal concircular transformations. Kodai Math. Sem. Rep. 1960, 12, 45-56. [CrossRef]

6. Fialkow, A. Conformal geodesics. Trans. Am. Math. Soc. 1939, 45, 443-473. [CrossRef]

7. Al-Dayel, I.; Deshmukh, S.; Belova, O. A remarkable property of concircular vector fields on a Riemannian Manifold. Mathematics 2020, 8, 469. [CrossRef]

8. $\quad$ O'Neill, B. Semi-Riemannian Geometry with Applications to Relativity; Academic Press: London, UK, 1983.

9. Takeno, H. Concircular scalar field in spherically symmetric spacetimes I. Tensor 1967, 20, 167-176.

10. Yano, K. On torse forming direction in a Riemannian space. Proc. Imp. Acad. Tokyo 1944, 20, 340-345. [CrossRef]

11. Chen, B.-Y. Rectifying submanifolds of Riemannian manifolds and torqued vector fields. Kragujev. J. Math. 2017, 41, 93-103. [CrossRef]

12. Duggal, K.L.; Sharma, R. Symmetries of Spacetimes and Riemannian Manifolds; Springer-Science + Busisness Media B.V.: Berlin, Germany, 1999.

13. Chaki, M.C.; Maity, R.K. On quasi Einstein manifolds. Publ. Math. Debr. 2000, 57, 297-306.

14. Besse, A.L. Einstein Manifolds; Springer: Berlin, Germany, 1987.

15. Hiepko, S. Eine innere Kennzeichnung der verzerrten Produkte. Math. Ann. 1979, 241, 209-215. [CrossRef]

16. Lee, J.M. Introduction to Smooth Manifolds; GMT-218; Springer: New York, NY, USA, 2003.

17. Fischer, A.E.; Marsden, J.E. Manifolds of Riemannian metrics with prescribed scalar curvature. Bull. Am. Math. Soc. 1974, 80, 479-484. [CrossRef] 